Article

\title{
Synthesis and Electro-Magneto-Mechanical Properties of Graphene Aerogels Functionalized with Co-Fe-P Amorphous Alloys
}

\author{
Guang-Ping Zheng *, Xi Lu and Zhuo Han \\ Department of Mechanical Engineering, Hong Kong Polytechnic University, Hung Hom, Kowloon, \\ Hong Kong, China; xi.lu@connect.polyu.hk (X.L.); zhuo.han@polyu.edu.hk (Z.H.) \\ * Correspondence: mmzheng@polyu.edu.hk; Tel.: +852-2766-6660 \\ Academic Editor: Hiroshi Mizuta \\ Received: 8 June 2016; Accepted: 5 July 2016; Published: 12 July 2016
}

\begin{abstract}
Graphene aerogels (GAs) are functionalized with Fe-Co-P alloy using an electro-deposition method. The Fe-Co-P alloy coated on the graphene nanosheets is found to possess an amorphous structure and a nanoporous architecture of GAs. The electro-mechanical properties of GAs are significantly affected by the Fe-Co-P nanoparticles embedded inside GAs. The electro-mechanical responses of GA/Fe-Co-P nanoporous hybrid structures are sensitive to an applied magnetic field, demonstrating that they are promising for electro-magneto-mechanical applications. The light-weight, high-strength and nanoporous GAs functionalized with Fe-Co-P amorphous alloys are desirable sensors, actuators, and nano-electro-mechanical systems that could be controlled or manipulated by mechanical, electric and magnetic fields.
\end{abstract}

Keywords: graphene aerogel; electro-magneto-mechanical properties; sensors; amorphous alloys

\section{Introduction}

Although graphene is a two-dimensional (2D) material with extraordinary properties, an immediate challenge in the applications of graphene is to develop bulk graphene architectures consisting of graphene nanosheets. Recently, three-dimensional (3D) graphene nanoporous structure, especially the graphene aerogel (GA), which is an aggregation network of 2D graphene, has attracted much attention [1-8]. Since GAs exhibit super-elasticity, high electro-mechanical sensitivity, ultra-light weight (density $\left.<1.0 \mathrm{mg} / \mathrm{cm}^{3}\right)$ and ultra-large surface area $\left(>200 \mathrm{~m}^{2} / \mathrm{g}\right)$, they are very promising to be used as devices and structures in micro-machines and nano-devices, such as sensors, actuators, and devices for energy storage and water purification [9-18].

Because of their exceptionally large surface areas (as large as $2630 \mathrm{~m}^{2} / \mathrm{g}$ ) and good electrical conductivity $\left(\sim 10^{2} \mathrm{~S} / \mathrm{m}\right)$, GAs are desirable supports for micro- and nano-sized metals, oxides, magnets, photo-catalysts and electro-catalysts, which have significantly enhanced performances compared with their pristine counterparts [9-18]. In particular, owing to their super-elasticity (up to $200 \%$ in compression) and stress-dependent electrical conductivity, GAs are suitable nano-electro-mechanical sensors and devices for various applications, such as micro- or nano-sized strain gauges, stress and pressure sensors. However, there are two major drawbacks of GAs which will prevent them from wide-spread applications. First, the high porosity of GAs usually results in significant reduction of their mechanical strength [1-4]. Second, the graphene nanosheets in GAs are reduced graphene oxides, which have low electrical conductivity [5-7]. Hence, graphene aerogel with improved mechanical and electrical performances are urgently needed.

In this work, we investigate GAs functionalized with Fe-Co-P alloys. The nanoporous structures of samples and the phases of Fe-Co-P embedded inside GAs are characterized. The mechanical 
and magnetic properties of the samples are measured. The relations between the electrical resistances of samples and the applied stresses and magnetic fields are determined, and the electro-magneto-mechanical applications of GA/Co-Fe-P hybrid structures are discussed.

\section{Experimental Methods}

Graphene oxides (GOs) were prepared by a modified Hummers method from natural graphite flakes. Graphene hydrogels (GHs) were fabricated via a hydrothermal process using GOs as the starting materials [2,3]. Firstly, $2 \mathrm{mg} \cdot \mathrm{mL}^{-1} \mathrm{GO}$ solution was hydrothermally treated in a Teflon-lined stainless-steel autoclave at $180^{\circ} \mathrm{C}$ for $20 \mathrm{~h}$ to obtain the GH. Ammonia solution was then added to the $\mathrm{GHs}$, which were subsequently treated in another autoclave at $90^{\circ} \mathrm{C}$ for $1 \mathrm{~h}$. After the hydrothermal treatment, the GHs were put in a freeze drying machine and were cooled to $-70{ }^{\circ} \mathrm{C}$. Supercritical drying of GHs occurred under a pressure of $10 \mathrm{kPa}$ for 2-3 days. Finally cylindrical graphene aerogels with a height of $\sim 15 \mathrm{~mm}$ were obtained. Those 3D graphene monoliths have a diameter of about 6-12 $\mathrm{mm}$.

The GA/Co-Fe-P hybrid structures were synthesized by electroplating Co-Fe-P alloys into the GAs. The $\mathrm{FeSO}_{4} \cdot 7 \mathrm{H}_{2} \mathrm{O}(4.171 \mathrm{~g}), \mathrm{CoSO}_{4} \cdot 7 \mathrm{H}_{2} \mathrm{O}(2.812 \mathrm{~g}), \mathrm{C}_{6} \mathrm{H}_{5} \mathrm{Na}_{3} \mathrm{O}_{7} \cdot 2 \mathrm{H}_{2} \mathrm{O}(14.705 \mathrm{~g}), \mathrm{H}_{3} \mathrm{BO}_{3}(7.729 \mathrm{~g})$, $\mathrm{NaH}_{2} \mathrm{PO}_{2} \cdot \mathrm{H}_{2} \mathrm{O}(5.3 \mathrm{~g})$ were dissolved into $250 \mathrm{~mL}$ deionized water to form an electrolyte. The $\mathrm{PH}$ value of the electrolyte was adjusted to $5-6$ using $\mathrm{H}_{2} \mathrm{SO}_{4}$. The temperature of the electrolyte was maintained at $60^{\circ} \mathrm{C}$. A GA with a height of about $5-10 \mathrm{~mm}$ was mounted on a titanium plate, which was used as a working electrode operating at a voltage of $1.0 \mathrm{~V}$ with respect to the reference electrode of saturated calomel electrode (SCE). The samples were electroplated for 2, 4, 8, and $14 \mathrm{~h}$, which were denoted as Sample-h2, h4, h8 and h14, respectively. After the electro-deposition the GAs were peeled off the titanium plate. The as-prepared GA/Co-Fe-P hybrid structures were then immersed into deionized water for two days to remove the electrolyte remained inside the GA. The samples were then dried by freeze drying at conditions of $-50^{\circ} \mathrm{C}$ and $20 \mathrm{~Pa}$ for $72 \mathrm{~h}$.

XRD patterns of the samples were taken by an X-ray diffractometer (Philips PW3040/60, Philips, Amsterdam, The Netherlands) with nickel filtered $\mathrm{Cu} \mathrm{K} \alpha$ radiation $(\lambda=0.154 \mathrm{~nm})$. Scanning Electron Microscopy (SEM, JEOL JSM-6490, JEOL Inc., Peabody, MA, USA) operated at $20 \mathrm{kV}$ was utilized to observe the microstructures of the samples. The SEM was equipped with energy dispersive X-ray (EDX, Peabody, MA, USA) analysis which was used to determine the samples' compositions. A surface area and porosity analyzer (Micromeritics ASAP 2420, Micromeritics Instrument Corporation, Norcross, GA, USA) was used to analyze the porous nature of the samples. The nitrogen adsorption-desorption isotherms were obtained at $-196{ }^{\circ} \mathrm{C}$ and the surface areas were calculated using the Barrett-Joyner-Halenda (BJH) method. Raman experiments were carried out on a Raman Station 400/400F with a resolution of $1.0 \mathrm{~cm}^{-1}$.

A mechanical testing machine (Materials Testing, ZWICK Z2.5 ${ }^{\mathrm{TH}}$, Zwick Techonology \& Instrument Co. Ltd., Shanghai, China) was used to measure the compressive strength of samples, which had been polished into cylindrical shape with a height of $5 \mathrm{~mm}$ and a diameter of 3-4 $\mathrm{mm}$. The compressive strain rate was $0.01 \mathrm{~min}^{-1}$. The electrodes of the samples were prepared by coating silver paste at their top and bottom surfaces. The currents of the sample under the applied voltages of 0-2 V were measured by the ferroelectric test system (TF2000E, aixACCT, Aachen, Germany) under the leakage current measuring mode. The electrical resistances of samples under compression were determined by the Ohm's law. The magnetic properties of the samples were characterized by the magnetic hysteresis loops, which were measured by a vibrating sample magnetometer (VSM, Lakeshore Model 7300, Lake Shore Cryotronics Inc., Westerville, OH, USA). The same procedures were applied to measure the electro-mechanical responses of the samples under an applied magnetic field which was supplied by a solenoid magnet.

\section{Results and Discussion}

\subsection{Characterizations of Samples}

SEM images of typical GAs and GA/Co-Fe-P samples are shown in Figure 1a,b. The shape of GA/Co-Fe-P sample is not much different with the cylindrical shape of monolith GA sample, 
as shown in the inset in Figure 1b. It can be seen in Figure 1 that the nanoporous architectures of GA are preserved in the GA/Co-Fe-P hybrid structures (Sample-h14) and the Co-Fe-P fully coated on the graphene nanosheets consists of a lot of nanoparticles. Figure 2 shows the SEM images of all GA/Co-Fe-P samples. The sizes and contents of Co-Fe-P nanoparticles in the hybrid structures can be found to increase with increasing deposition time. It seems that the Co-Fe-P alloys fully coated on the graphene nanosheets in Sample-h14 could result from the growth of Co-Fe-P nanoparticles whose sizes can be as small as 20-30 nm, as shown in Figure 2a for Sample-h2.
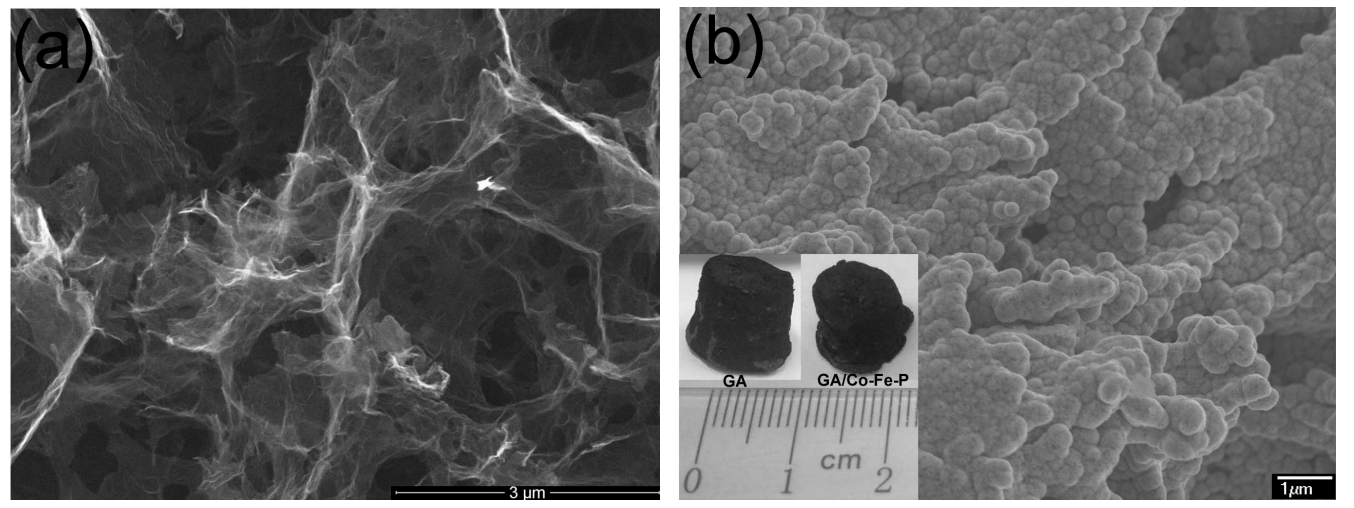

Figure 1. SEM images of GA (a) and GA/Co-Fe-P (Sample-h14) (b). The inset in (b) shows the typical size and shape of GA and GA/Co-Fe-P samples.
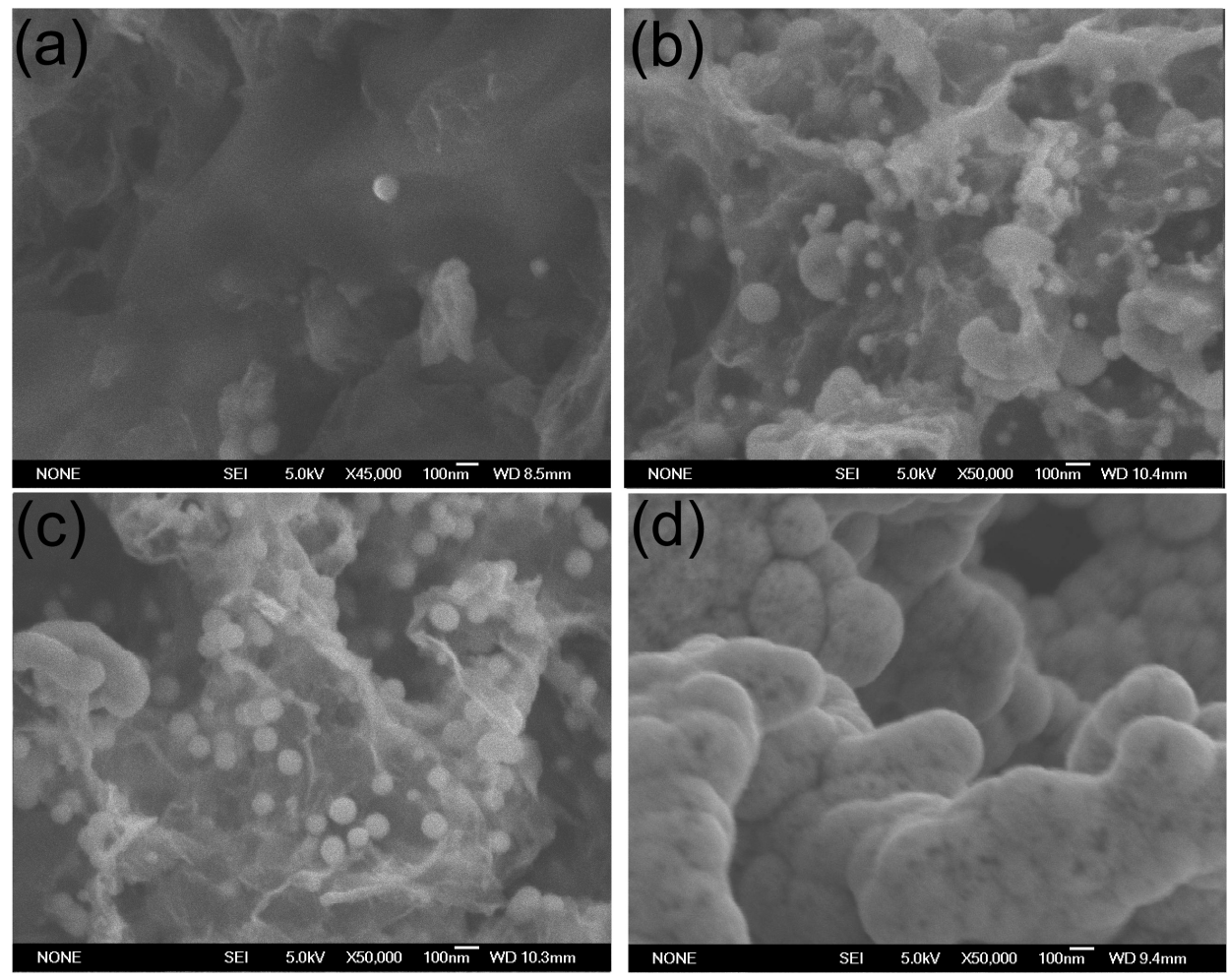

Figure 2. SEM images of GA/Co-Fe-P samples: (a) Sample-h2; (b) Sample-h4; (c) Sample-h8; (d) Sample-h14.

Figure 3 shows the XRD patterns of GA/Co-Fe-P samples. In comparison with those of GA and Co-Fe-P foils prepared by the same electrodeposition conditions, the XRD peaks for Sample-h14 demonstrate that the Co-Fe-P alloys coated on the graphene nanosheets is amorphous, as shown in 
Figure $3 \mathrm{~b}$. The intensity of the broad peak at $44.7^{\circ}$ for the amorphous phase of Co-Fe-P indicates that its content dramatically increases when the electrodeposition time is larger than $8 \mathrm{~h}$, as shown in Figure 3a for Sample-h2, h4 and h8 and in Figure 3b for Sample-h14.
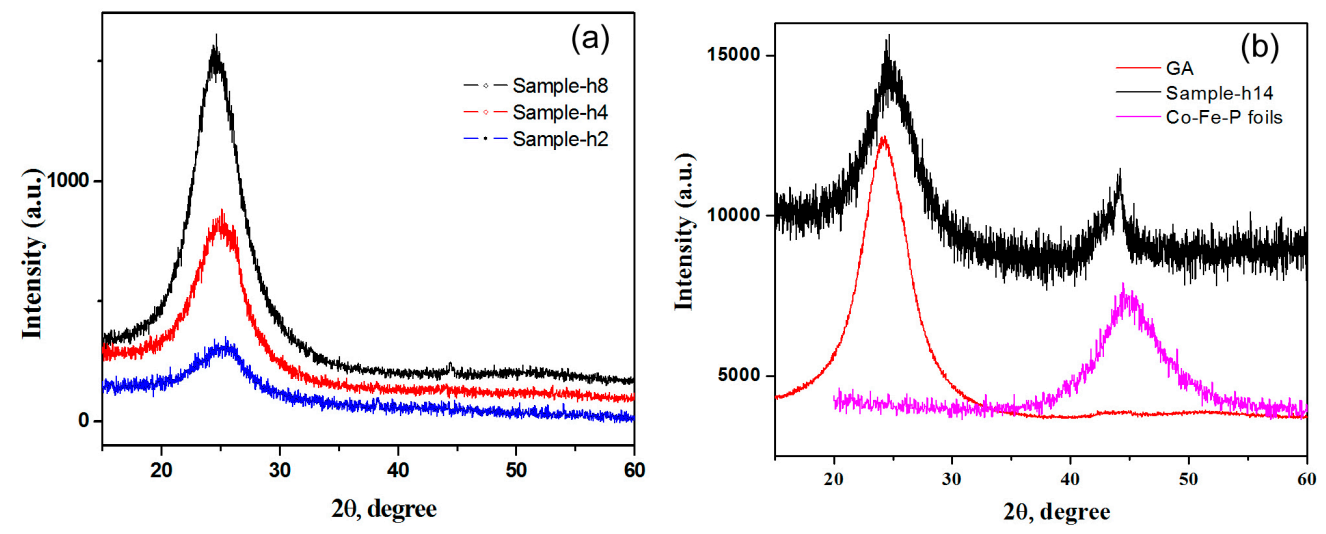

Figure 3. XRD patterns of GA and GA/Co-Fe-P samples: (a) Sample-h2, Sample-h4 and Sample-h8; (b) GA, Co-Fe-P foils and Sample-h14.

Table 1 lists the results of analysis on the nanoporous structures of GA/CO-Fe-P samples. As indicated by the specific surface area (SSA) and volume $\left(\mathrm{V}_{\text {total }}\right)$, the Co-Fe-P nanoparticles embedded on the graphene nanosheets significantly reduce the SSA and $V_{\text {total }}$ of Sample-h2 and Sample-h4. With the evolution of Co-Fe-P nanoparticles throughout the graphene nanosheets, the SSA and $V_{\text {total }}$ of Sample-h8 and Sample-h14 increase, suggesting that the Co-Fe-P amorphous alloy tends to form film-like coating on the surfaces of graphene nanosheets when the content of Co-Fe-P increases. As listed in Table 1, the average size of nano-pores of the hybrid structures increases with increasing content of Co-Fe-P.

Table 1. The composition, specific surface area (SSA) and volume $\left(\mathrm{V}_{\text {total }}\right)$, and pore width of GA and GA/Co-Fe-P samples.

\begin{tabular}{ccccc}
\hline Samples & Co/Fe/P Mole Fraction & SSA $\left(\mathbf{m}^{\mathbf{2}} \cdot \mathbf{g}^{-\mathbf{1}}\right)$ & $\mathbf{V}_{\text {total }} \mathbf{( \mathbf { c m } ^ { \mathbf { 3 } } \mathbf { g } )}$ & Pore Width $(\mathbf{n m})$ \\
\hline GA & - & 370.9022 & 0.449374 & 5.81898 \\
Sample-h2 & - & 70.225 & 0.071297 & 4.06106 \\
Sample-h4 & - & 10.7219 & 0.020687 & 7.71771 \\
Sample-h8 & $32.5 / 32.7 / 33.8$ & 44.9051 & 0.11327 & 10.08971 \\
Sample-h14 & $31.2 / 25.7 / 43.1$ & 55.5464 & 0.154443 & 11.12175 \\
\hline
\end{tabular}

Raman spectra of the GA/Co-Fe-P samples are shown in Figure 4. The D-band $\left(1327 \mathrm{~cm}^{-1}\right)$ represents the defects in the graphene or amorphous carbon; the G-band $\left(1593 \mathrm{~cm}^{-1}\right)$ is caused by the in-plane vibration of graphite with an $\mathrm{E}_{2 \mathrm{~g}}$-symmetry intra-layer mode. The intensity ratio of $\mathrm{D}$-band to G-bands $\left(\mathrm{I}_{\mathrm{d}} / \mathrm{I}_{\mathrm{g}}\right)$ is higher in Sample-h2 than those of any other samples, suggesting that the defective carbon structures can be induced in Sample-h2 where Co-Fe-P nanoparticles with sizes of 20-30 nm might interact with the graphene nanosheets or their junctions. Becasue the surfaces of Co-Fe-P nanoparticles are less active toward the bonding with carbon atoms of the graphene nanosheets when the sizes of nanoparticles are increased, therefore the ratio $I_{d} / I_{g}$ could decrease to that of graphene with increasing sizes of nanoparticles. 


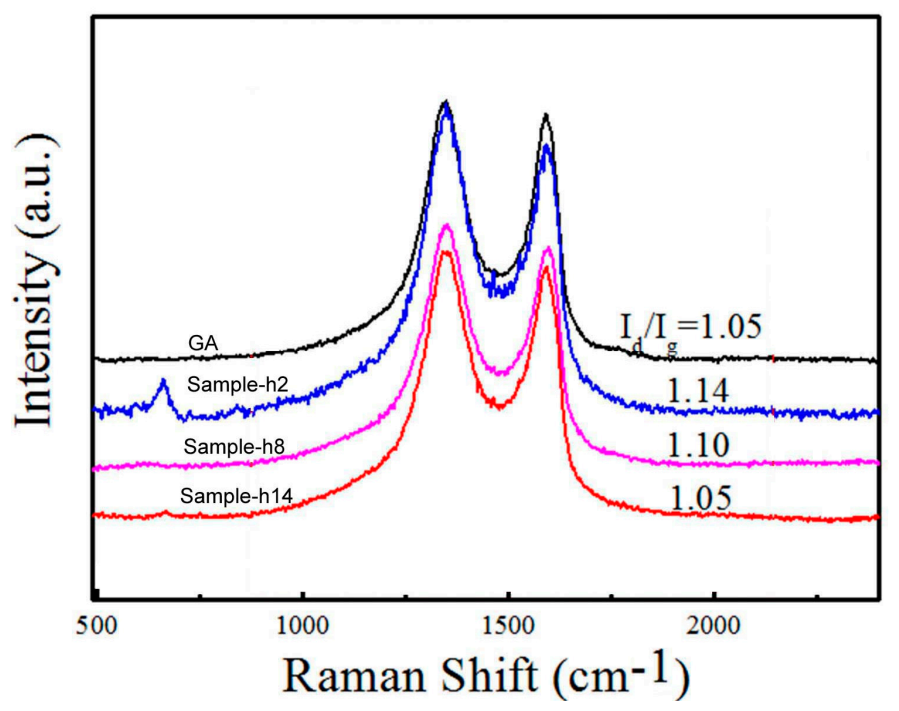

Figure 4. Raman spectra of GA and GA/Co-Fe-P samples. $I_{d} / I_{g}$ is the ratio of intensities of Dand G-bands.

\subsection{The Electro-Mechanical Properties of GA/Co-Fe-P Samples}

The compressive strength of Sample- $\mathrm{h} 2, \mathrm{~h} 4, \mathrm{~h} 8$ and $\mathrm{h} 14$ are determined from the compression tests to be $0.2,3.8,22$ and $620 \mathrm{kPa}$, respectively. Except for Sample-h2 whose fracture strength is smaller than that of GA $(0.6 \mathrm{kPa})$, the GA/Co-Fe-P samples exhibit much improved strength as compared with the monolith GA. The small mechanical strength of Sample-h2 might be related with the disorder graphene nanosheets induced by the embedded Co-Fe-P nanoparticles with sizes of 20-30 nm. When the Co-Fe-P amorphous alloys fully coat the graphene nanosheets inside GA, as shown in Figure 1b for Sample-h14, the mechanical properties of GA/Co-Fe-P hybrid structure are mainly determined by the Co-Fe-P amorphous alloys. Therefore the fracture strength of GA/Co-Fe-P hybrid structure increases with the increasing content of $\mathrm{Co}-\mathrm{Fe}-\mathrm{P}$ in a non-linear manner.

The GA/Co-Fe-P hybrid structures are placed along the horizontal direction between two copper plates. Thin copper wires are attached to the two copper plates which are separately contacted with the top and bottom electrodes of the samples. The copper plates are driven to move horizontally in the opposite directions, leading to the compression on the samples. When the samples are compressed by a uniaxial compressive strain $\varepsilon$, the electrical resistances $R(\varepsilon)$ of the samples are measured. Figure 5 shows the repeatable change of electrical resistance $\Delta R=|R-R(\varepsilon)|$ of the GA/Co-Fe-P samples (Sample-h4 and Sample-h8) with $\varepsilon$, which is cyclic between 0 and $\varepsilon_{\mathrm{m}}$. R is the electrical resistance at $\varepsilon=0$. A linear relation between $\Delta R / R$ and $\varepsilon$ changing from 0 to $\varepsilon_{\mathrm{m}}$ and then back to 0 can be observed, the $\Delta R / R \sim \varepsilon$ relations for Sample-h2 and Sample-h14 are not shown either because they have $\varepsilon_{\mathrm{m}}<2 \%$ (Sample-h2) or the change in $\Delta \mathrm{R} / \mathrm{R}$ is less than $1 \%$ (Sample-h14).

The repeatable changes of electrical resistances under applied strains in Sample-h4 and Sample-h8 indicate that they have better electro-mechanical performances or sensitivities compared to monolith GA. Combined with its high mechanical strength and light weight, Sample-h8 has the best performance in nano-electro-mechanical applications.

\subsection{The Electro-Magneto-Mechanical Properties of GA/Co-Fe-P Samples}

The electro-deposited Co-Fe-P alloy foils have been well studied to have excellent soft magnetic properties [19]. Although in the GA/Co-Fe-P hybrid structures only Sample-h14 is found to exhibit magnetic properties which are detectable by the VSM, as shown in Figure 6a, other GA/Co-Fe-P hybrid structures containing Fe-Co-P magnetic materials could be also sensitive to an applied magnetic 
field. Therefore, the repeatable changes of electrical resistances of the GA/Co-Fe-P samples under applied strains could be affected by the applied magnetic fields.

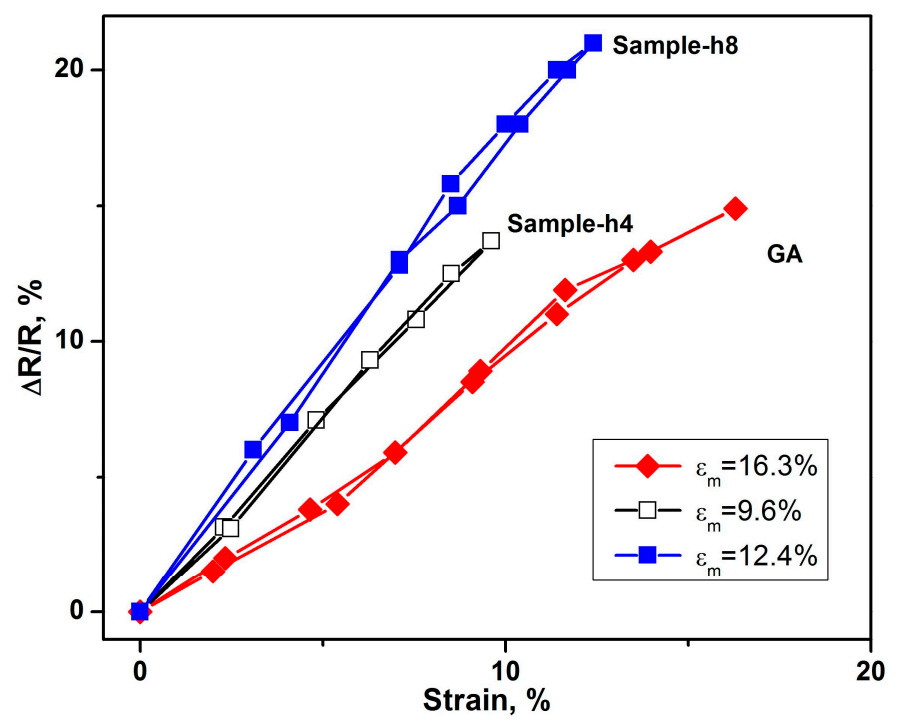

Figure 5. The relative changes of resistances of GA and GA/Co-Fe-P samples under compressive strains in a loading (from 0 to $\varepsilon_{\mathrm{m}}$ ) and un-loading (from $\varepsilon_{\mathrm{m}}$ to 0 ) cycle. $\varepsilon_{\mathrm{m}}$ denotes the maximum applied strain.
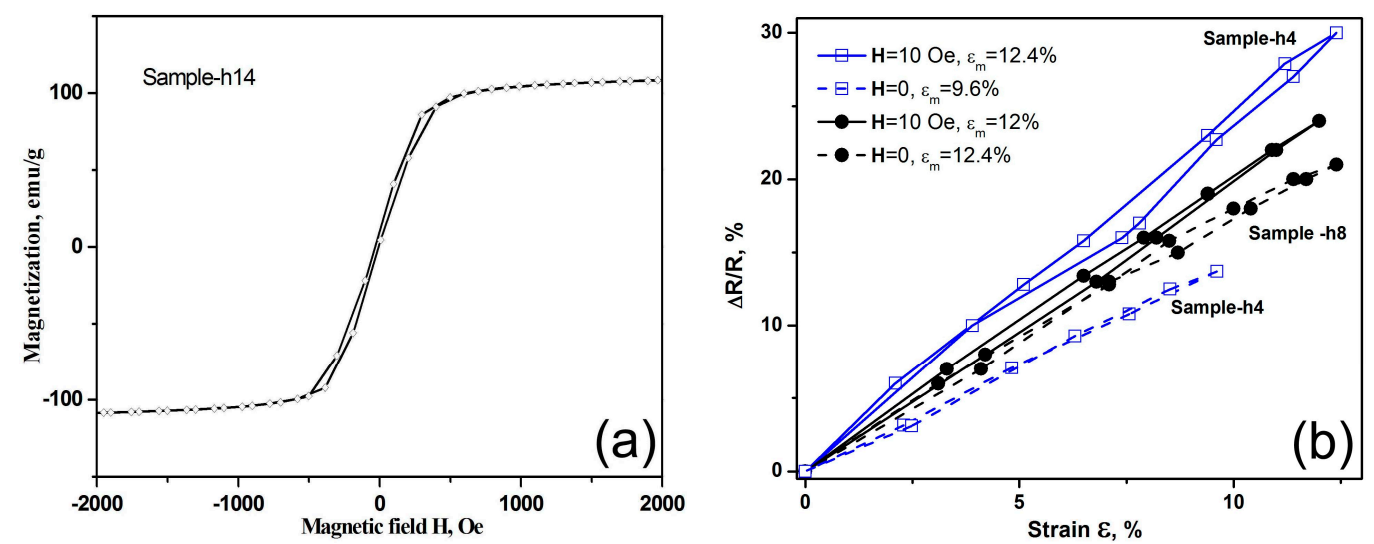

Figure 6. (a) Magnetic hysteresis loop of the GA/Co-Fe-P sample; (b) The relative changes of resistances of GA/Co-Fe-P samples under compressive strains in a loading (from 0 to $\varepsilon_{\mathrm{m}}$ ) and un-loading (after $\varepsilon_{\mathrm{m}}$ to 0 ) cycle, with and without an applied magnetic field $H$. $\varepsilon_{\mathrm{m}}$ denotes the maximum applied strain.

Figure $6 \mathrm{~b}$ shows the $\Delta \mathrm{R} / \mathrm{R} \sim \mathcal{\varepsilon}$ relations for Sample-h4 and Sample-h8 with and without a magnetic field of 10 Oe applied along the compression direction. Both samples have enhanced electro-mechanical sensitivity when a magnetic field is applied. Remarkably, the electro-mechanical responses under a magnetic field can be as high as 3 times of those of Sample-h4 without an applied magnetic field. Although the effects of nanoporous structures of GA/Co-Fe-P and the sizes of Co-Fe-P nanoparticles embedded on the graphene nanosheets on the electro-magneto-mechanical properties are yet to be further explored, the results suggest that the GA/Co-Fe-P hybrid structures have outstanding electro-magneto-mechanical responses and could be promising nano-electro-mechanical systems controlled or manipulated by multiple applied fields. 


\section{Conclusions}

We investigate GAs functionalized with Fe-Co-P alloys, which are promising materials for nano-electro-mechanical systems and nano-devices. The GA/Co-Fe-P hybrid structures synthesized by electrodeposition are found to have nanoporous structures and contain amorphous Fe-Co-P nanoparticles embedded on the graphene nanosheets. The relations between the electrical resistances of samples and the applied strains and magnetic fields are determined. The results demonstrate that GAs functionalized with Fe-Co-P amorphous alloys are suitable for electro-magneto-mechanical applications in micro-machine and nano-devices.

Acknowledgments: The authors are grateful for the support provided by the Science and Technology Innovation Commission of Shenzhen, China (No. JCYJ2013041152508657).

Author Contributions: Guang-Ping Zheng designed the experiments; Xi Lu, Zhuo Han and Guang-Ping Zheng performed the experiments; Zhuo Han and Guang-Ping Zheng analyzed the data; Guang-Ping Zheng wrote the paper.

Conflicts of Interest: The authors declare no conflict of interest.

\section{References}

1. Novoselov, K.S.; Geim, A.K.; Morozov, S.V.; Jiang, D.; Zhang, Y.; Dubonos, S.V.; Grigorieva, I.V.; Firsov, A.A. Electric field effect in atomically thin carbon films. Science 2004, 306, 666. [CrossRef] [PubMed]

2. Hu, H.; Zhao, Z.; Wan, W.; Gogotsi, Y.; Qiu, J. Ultralight and Highly Compressible Graphene Aerogels. Adv. Mater. 2013, 25, 2219-2223. [CrossRef] [PubMed]

3. Sun, H.; Xu, Z.; Gao, C. Multifunctional, Ultra-Flyweight, Synergistically Assembled Carbon Aerogels. Adv. Mater. 2013, 25, 2554-2560. [CrossRef] [PubMed]

4. Ito, Y.; Tanabe, Y.; Qiu, H.J.; Sugawara, K.; Heguri, S.; Tu, N.H.; Huynh, K.K.; Fujita, T.; Takahashi, T.; Tanigaki, K.; et al. High-Quality Three-Dimensional Nanoporous Graphene. Angew. Chem. 2014, 126, 4922-4926. [CrossRef]

5. Biener, J.; Dasgupta, S.; Shao, L.; Wang, D.; Worsley, M.A.; Wittstock, A.; Lee, J.R.; Biener, M.M.; Orme, C.A.; Kucheyev, S.O.; et al. Macroscopic 3D nanographene with dynamically tunable bulk properties. Adv. Mater. 2012, 24, 5083-5087. [CrossRef] [PubMed]

6. Feng, W.; Yan, L. In situ Self-assembly of Mild Chemical Reduction Graphene for Three-dimensional Architectures. Nanoscale 2011, 3, 3132-3137.

7. Worsley, M.A.; Kucheyev, S.O.; Satcher, J.H., Jr.; Hamza, A.V.; Baumann, T.F. Mechanically robust and electrically conductive carbon nanotube foams. Appl. Phys. Lett. 2009, 94, 073115. [CrossRef]

8. Kim, K.H.; Oh, Y.; Islam, M.F. Graphene coating makes carbon nanotube aerogels superelastic and resistant to fatigue. Nat. Nanotechnol. 2012, 7, 562. [CrossRef] [PubMed]

9. Zhang, X.; Sui, Z.; Xu, B.; Yue, S.; Luo, Y.; Zhan, W.; Liu, B. Mechanically strong and highly conductive graphene aerogel and its use as electrodes for electrochemical power sources. J. Mater. Chem. 2011, $21,6494$. [CrossRef]

10. Zhou, G.; Yin, L.-C.; Wang, D.-W.; Li, L.; Pei, S.; Gentle, I.R.; Li, F.; Cheng, H.-M. Fibrous Hybrid of Graphene and Sulfur Nanocrystals for High-Performance Lithium Sulfur Batteries. ACS Nano 2013, 7, 5367-5375. [CrossRef] [PubMed]

11. Hao, G.-P.; Hippauf, F.; Oschatz, M.; Wisser, F.M.; Leifert, A.; Nickel, W.; Mohamed-Noriega, N.; Zheng, Z.; Kaskel, S. Stretchable and Semitransparent Conductive Hybrid Hydrogels for Flexible Supercapacitors. ACS Nano 2014, 8, 7138. [CrossRef] [PubMed]

12. Choi, B.G.; Yang, M.; Hong, W.H.; Choi, J.W.; Huh, Y.S. 3D Macroporous Graphene Frameworks for Supercapacitors with High Energy and Power Densities. ACS Nano 2012, 6, 4020-4028. [CrossRef] [PubMed]

13. Zhang, L.; Zhang, F.; Yang, X.; Long, G.; Wu, Y.; Zhang, T.; Leng, K.; Huang, Y.; Ma, Y.; Yu, A.; et al. Porous 3D graphene-based bulk materials with exceptional high surface area and excellent conductivity for supercapacitors. Sci. Rep. 2013, 3, 1408. [CrossRef] [PubMed]

14. Gong, Y.; Yang, S.; Liu, Z.; Ma, L.; Vajtai, R.; Ajayan, P.M. Graphene-Network-Backboned Architectures for High-Performance Lithium Storage. Adv. Mater. 2013, 25, 3979-3984. [CrossRef] [PubMed] 
15. Xiao, L.; Wu, D.; Han, S.; Huang, Y.; Li, S.; He, M.; Zhang, F.; Feng, X. Self-Assembled $\mathrm{Fe}_{2} \mathrm{O}_{3} / \mathrm{Graphene}$ Aerogel with High Lithium Storage Performance. ACS Appl. Mater. Interfaces 2013, 5, 3764-3769. [CrossRef] [PubMed]

16. Hodlur, R.M.; Rabinal, M.K. Self Assembled Graphene Layers on Polyurethane Foam as a Highly Pressure Sensitive Conducting Composite. Compos. Sci. Technol. 2014, 90, 160-165. [CrossRef]

17. Kuang, J.; Liu, L.; Gao, Y.; Zhou, D.; Chen, Z.; Han, B.; Zhang, Z. A hierarchically structured graphene foam and its potential as a large-scale strain-gauge sensor. Nanoscale 2013, 5, 12171-12177. [CrossRef] [PubMed]

18. Huang, H.; Chen, P.; Zhang, X.; Lu, Y.; Zhan, W. Edge-to-Edge Assembled Graphene Oxide Aerogels with Outstanding Mechanical Performance and Superhigh Chemical Activity. Small 2013, 9, 1397-1404. [CrossRef] [PubMed]

19. Huang, W.-Q.; Huang, G.-F.; Liang, B.; Xie, C.-L. Magnetic properties of CoFeP films prepared by electroless deposition. J. Magn. Magn. Mater. 2009, 321, 1177-1181. [CrossRef]

(C) 2016 by the authors; licensee MDPI, Basel, Switzerland. This article is an open access article distributed under the terms and conditions of the Creative Commons Attribution (CC-BY) license (http://creativecommons.org/licenses/by/4.0/). 